\title{
Global database of direct solar radiation at the Moon's surface for lunar engineering purposes
}

\author{
Marcin Kaczmarzyk ${ }^{1, *}$, Marcin Gawronski ${ }^{2}$, Grzegorz Piatkowski ${ }^{3}$ \\ ${ }^{1}$ Department of building engineering, Rzeszow University of Technology, Poznanska 2 35-959 \\ Rzeszow, Poland \\ ${ }^{2}$ Centre for Astronomy, Faculty of Physics, Astronomy and Informatics, Nicolaus Copernicus \\ University, Grudziadzka 5, 87-100 Torun, Poland \\ ${ }^{3}$ Department of Structural Mechanics, Rzeszow University of Technology, Poznanska 2 35-959 \\ Rzeszow, Poland
}

\begin{abstract}
The purpose of this paper was to provide preliminary data concerning global availability of solar energy at the surface of the Moon. Lack of gaseous atmosphere and accompanying phenomena such as precipitations or cloud cover makes the Moon's surface an extraordinarily advantageous place for solar energy harvesting. On the other hand, excessive exposure to undamped sunlight may cause problems with buildings' interior overheating or increase decay rate of photovoltaic cells. Thus, basic information concerning solar irradiance and diurnal insolation at specified selenographic latitudes are indispensable for location selection for future lunar facilities and their design process. In order to approximate Sun's position at lunar sky, simple analytical astrometric model of lunar rotation was developed. Basing on that model, direct diurnal irradiances and insolations were calculated for various flat surface orientations, and selenographic latitudes. Computed data were presented in charts and tables.This lunar insolation database may serve as guideline for location of future lunar settlements and research stations, and to estimate their diurnal energy demands.
\end{abstract}

\section{Introduction}

\subsection{Solar energy and earth-Moon system}

Energy emitted by the sun is produced by nuclear fusion reactions, that take place in its core. It is estimated, that every second about 4.26 million metric tons of the Sun's mass is being converted into energy, what results in total radiant power of the Sun being $3.828 \times 10^{26} \mathrm{~W}[1]$. This power radiates uniformly into space, so, for a surface perpendicular to the incident sunlight, radiant flux per unit area is inversely proportional to the square of the distance from the centre of the Sun, according to equation (1) [2]:

\footnotetext{
${ }^{*}$ Corresponding author: kaczmar@prz.edu.pl
} 


$$
T S I=\frac{L_{\text {sol }}}{4 \pi \cdot R^{2}}\left[\frac{W}{m^{2}}\right]
$$

Equation (1): Total solar irradiance (TSI) as a function of distance to the Sun, where:

$\mathrm{L}_{\text {sol }}$ - solar luminosity $3.828 \times 10^{26}[\mathrm{~W}]$,

$\mathrm{R}$ - distance to the Sun's centre [m].

TSI calculated at one astronomical unit from the Sun i.e. mean Earth-Sun distance equals $1,361 \mathrm{~W} / \mathrm{m}^{2}$ and is often referred as solar constant. As the Sun's activity varies slightly in time, solar constant is not an absolute constant in a scientific sense, rather a mean value.

Due to slight Eccentricity of Earth's orbit, instantaneous values of TSI above earth's atmosphere varies annually between $1,412 \mathrm{~W} / \mathrm{m}^{2}$ at perihelion and $1,320 \mathrm{~W} / \mathrm{m}^{2}$ at aphelion [3].

Insolation, or radiant exposure, is an integral of solar irradiance over a specified period of time, i.e. it is the sum of energy of full-spectrum solar electromagnetic radiation that reaches specifically oriented surface in a specified period of time, per unit area. Direct insolation may be calculated as shown by equation (2) [4].

$$
I=\int_{t_{0}}^{t_{1}} E_{d i r}(t) d t\left[\frac{J}{m^{2}}\right]
$$

Equation (2): Direct insolation of a specified surface for time period $t_{0}-t_{1}$, where:

$t_{0}$ and $t_{1}$ is considered period of time,

$\mathrm{E}_{\mathrm{dir}}$ is instantaneous direct solar irradiance i.e. intensity of direct solar radiation at a specified surface $\left[\mathrm{W} / \mathrm{m}^{2}\right]$.

\subsection{Lunar orbit}

The Moon completes single geocentric orbit relative to distant stars (sidereal period) in 27.322 days. Due to its tidal locking, The Moon exhibits almost perfect synchronous rotation i.e. its rotational period equals its sidereal period, and that is why the Moon always faces Earth with almost the same side. Such slow rotation combined with Earth-Moon system orbital motion lengthens Moon's rotational period relative to the Sun (synodic month) to mean value of 29.531 days [5]. Moon's axial tilt with respect to the ecliptic is only $1,54^{\circ}$, so any effects of lunar seasons are negligible for most practical considerations [6, 7].

Because there exists no orbital resonance between Moon's and earth's orbit, Moon's instantaneous distance to Earth quasiperiodically influences its distance to the Sun up to 768 thousand kilometres [5].

The Moon's synchronous rotation causes that the moon's far side is closer to the Sun during local day than Moon's near side. This effect is most pronounced for $0^{\circ}$ and $180^{\circ}$ selenographic longitude.

In that case, putting specified combinations of earth's and Moons apsidal distances into equation(1) it is possible to calculate annual extreme TSI values at the Moon's surface and depict them in table 1 . 
Table 1. Extreme values of TSI at the Moon's surface calculated for selected orbital positions of the Earth and the Moon.

\begin{tabular}{|c|c|c|c|}
\hline \multicolumn{2}{|c|}{ Orbital positions } & \multicolumn{2}{c|}{ Total solar irradiance $\left[\mathbf{W} / \mathbf{m}^{\mathbf{2}}\right]$} \\
\hline Earth & Moon & near side $\left(\mathbf{0}^{\circ}\right.$ longitude) & Far side (180 ${ }^{\circ}$ longitude) \\
\hline perihelion & perigee & $1,401.65$ & $1,415.54$ \\
\hline perihelion & apogee & $1,400.83$ & $1,416.36$ \\
\hline aphelion & perigee & $1,311.18$ & $1,323.74$ \\
\hline aphelion & apogee & $1,310.44$ & $1,324.49$ \\
\hline
\end{tabular}

\subsection{Lunar surface environment}

Physical conditions at the Moon's surface differ significantly from those on Earth. The Moon has no gaseous atmosphere, but very sparse exosphere of pressure about $0,3 \mathrm{nPa}$ [5] which may be considered as perfect vacuum for any engineering purposes. Hydrosphere does not exist on the Moon as well, although existence of water ice has been confirmed in permanently shadowed craters at lunar poles [8]. Unprotected by atmosphere nor magnetosphere, lunar surface is exposed to cosmic radiation. Surface of the Moon is covered by stratum of crushed and pulverised rock, called regolith. Due to its low thermal inertia, lunar surface remains in near thermal equilibrium with incident solar radiation [9], The regolith, especially its thin, uppermost $2 \mathrm{~cm}$ stratum has extremely low thermal conductivity. This property, along with low bulk density of surface material causes very high near-surface temperature gradients in lunar regolith. Very little heat penetrates deeper than $50 \mathrm{~cm}$ below lunar surface, and below 80-90 cm temperature remains constant at specified depth, resulting only from the Moon's internal heat flux [10].

\subsection{Selected issues of lunar engineering}

Up to this date, lunar engineering remains mostly theoretical field. Several analogue bases, so called habitats, were built in order to study mostly psychosocial aspects of future lunar and martian manned missions [11].

As a part of in situ resource utilization strategy, lunar regolith samples and their analogs were studied for potential applications as structural and thermal insulating materials [12-15]. Several works were dedicated to create basic guidelines for lunar architecture and colony development strategies [16]. Most of these assume using raw or processed regolith for massive thermal and radiation shielding envelopes for long-term habitation. Before we reach that level of development, some equipment and astronauts must perform their task in much less friendly conditions, especially being exposed to direct solar radiation. During further development, reliable data concerning solar irradiance and insolation will be necessary for designing transparent building barrier's for habitats and agricultural centres. These data will also be indispensable for photovoltaic power plants and potentially for some specialised industries.

Atmospheric mass at the Moon's surface always equals zero, no matter the time of day. In that case, that at any latitude and for any positive elevation of the Sun, one may find thus oriented surface, that receives maximum possible value of TSI. This makes solar radiation a promising energy source for future lunar facilities.

On the other hand, excessive sunlight exposure causes problems with overheating buildings interiors even in moderate climate zones on earth $[17,18]$. This problem may become a much serious issue at the Moon's surface, especially when limited heat exchange in hard vacuum is considered. Increased degradation rate of photovoltaic cells in space is mainly caused by high energy particles from space radiation, but also by prolonged 
exposure to high temperatures [19]. One may conclude, that lunar surface presents extraordinarily advantageous conditions for solar energy harvesting, as long as all the aspects of local illumination are taken under consideration.

On Earth, monthly Solar irradiation data are indispensable for civil engineers and architects to calculate thermal balance of a building and to determine its energy demand. Because earth's surface environment is far more complex than the Moon's, these data cannot be calculated, but are based on long-term measurements. In the contrary, lack of atmospheric phenomena causes, that illumination of the Moon's surface is not subjected to any stochastic variations, is periodically repetitive, and may be accurately determined as a function of time and selenographic latitude.

The purpose of this study is to provide analogical set of preliminary data for selected selenographic latitudes.

\section{Model description}

\subsection{General assumptions}

This paragraph describes the method we applied to calculate solar irradiance and insolations for various selenographic latitudes. As only the global preliminary data are sought, our calculations does not consider site shadowing by terrain features, nor diffuse radiation, but direct solar radiation only.

Because the moon's surface experiences about $4 \%$ annual TSI variations (see 1.2.), a mean TSI value of $1363.03 \mathrm{~W} / \mathrm{m}^{2}$ was assumed for the our analysis. We conducted the calculations for northern hemisphere, between $0^{\circ}$ and $80^{\circ}$ latitudes with $10^{\circ}$ intervals. Higher latitudes were not studied due to high influence of local terrain topography on the illumination for low Sun altitudes. Obtained data are symmetrical with respect to the equator. The dataset covers four cardinal directions, with five tilt values each, presented in horizontal coordinate system.

Solar irradiance

Direct solar irradiance for a flat surface of specified orientation (tilt and azimuth) as a function of time may be calculated as [2]:

$$
E_{d i r}(t)=T S I \cdot \cos (\delta(t))\left[\frac{W}{m^{2}}\right]
$$

Equation (3): Instantaneous solar irradiance for a flat surface,

where $\delta$ is an angle of incidence for solar radiation as a function of time $t$, that passed since local dawn.

Insolation (radiant exposure)

By definition, insolation is calculated according to equation (2). For a lunar the day, this expression may be approximated by following formula:

$$
I=\sum_{0}^{T_{\text {svn }} / 2} E_{d i r}(t) \cdot \Delta \tau\left[\frac{J}{m^{2}}\right]
$$

Equation (4): Approximated formula for determining insolation value, where 0 and $\mathrm{t}_{\mathrm{syn}} / 2$ are moments of lunar local dawn and sunset respectively $\Delta \tau$ is a time step

If a time step is sufficiently short, equation (4) yields highly accurate results. 
Numerous engineering concepts of lunar bases considers hemispherical shape of the building as the most beneficial solution [16]. Due to this fact, we found it purposeful to calculate daily insolations for 10 metres in diameter reference hemisphere.

Total amount of energy of solar radiation reaching a surface of a hemisphere may be calculated as:

$$
E_{h s}(t)=\sum_{0}^{T_{s y n} / 2} A_{h s}(t) \cdot T S I[J]
$$

Equation (5): Total solar radiant energy reaching a hemispherical surface, where $A_{h s}$ is a surface area of a parallel projection of a hemisphere onto a plane perpendicular to direction of the incoming sunlight. In a general case, such projection of a hemisphere produces a combination of a semicircle and a semi-ellipse. The semicircle has a constant radius $\mathrm{r}_{\mathrm{hs}}$, equal to one of a hemisphere.

A semi-major axis a of semi-ellipse is also constant, being equal to hemisphere's radius. $A=r_{h s}$

Length of the Semi-minor axis b is variable and may be expressed as:

$B=r_{h s} \cdot \sin \left(H_{\text {sol }}(t)[m]\right.$

where $\mathrm{H}_{\text {sol }}(\mathrm{t})$ is an instantaneous solar altitude.

As an ellipse's surface area equals $\pi \cdot \mathrm{A} \cdot \mathrm{B}$, total surface area of hemisphere's parallel projection may be calculated according to equation (6):

$$
\left.A_{h s}(t)=0,5 \cdot \pi \cdot r_{h s}^{2} \cdot\left(1+\sin \left(H_{s o l}(t)\right)\right)\right)\left[m^{2}\right]
$$

All three formulae (3), (4) and (5) require instantaneous positions of the Sun in horizontal coordinate system as an input data. For this purpose astronomers use adequate ephemeris tables or special computer programs. We found both of these solutions inconvenient for engineering purposes. An attempt was made to create a simple, analytical model of the Moon's rotation, that would provide an engineer necessary data with required accuracy. It was found, that properties of the Moon's orbit presents an opportunity to create such a simple, yet still sufficiently accurate model.

\subsection{Analytical model of the Moon's rotation}

The Earth and its natural satellite - the Moon constitute a complex, dynamical system. Hence, the determination of the accurate ephemerides for selected celestial bodies on the Moon's sky is a non-trivial problem, and complicated mathematical formulae are needed to be used. However, it is possible to build a simple model, that will allow to estimate Sun's position in the lunar sky with precision of $1-2^{\circ}$, what is fully acceptable for engineering purposes.

The plane of the lunar orbit is inclined to the ecliptic by about $5.15^{\circ}$, and the rotational axis of the Moon is inclined to its orbital axis by $6.69^{\circ}$ [5]. In 1722 Giovanni Domenico Cassini discovered, that the rotational axis of the Moon precesses with the same rate as its orbital plane, but is shifted by $180^{\circ}$ in phase [5]. Therefore, the angle between the ecliptic and the lunar equator remains almost constant $\left(1.54^{\circ}\right)$. For the simplicity of the model it may be assumed, that the equatorial plane of the Moon is coplanar with the ecliptic plane, i.e. the rotational axis of the Moon is perpendicular to the ecliptic plane. Moreover, it may also be assumed, that the Moon is located in the barycenter of the Earth-Moon system. The radius of lunar orbit is significantly smaller than the radius of the Earth's orbit, hence all effects caused by the trigonometric parallax of the Sun on the Moon's firmament may be neglected in this model. To summarise, proposed model of the Earth-Moon system could be reduced to the Moon located in the Earth's orbit with the rotational axis perpendicular to the ecliptic plane. 


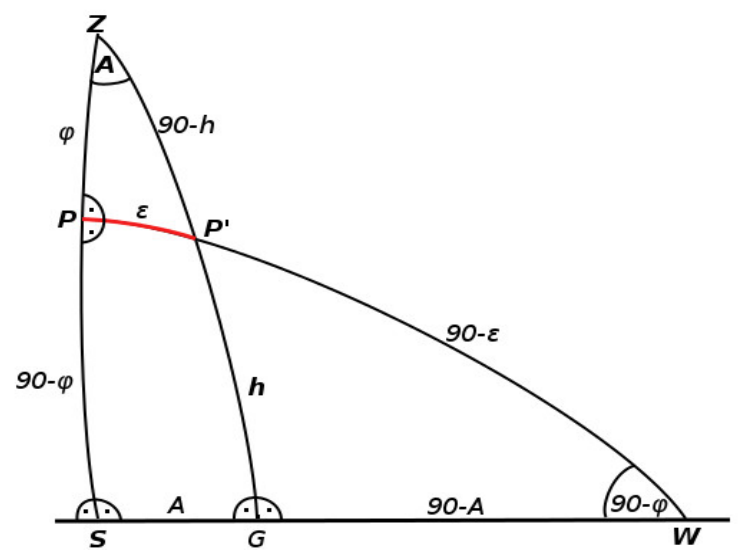

Fig. 1. Graphic presentation of proposed simple astrometric model for estimation of the azimuth and elevation angle of the Sun for a given time and location on the Moon's surface. $\mathbf{Z}$-zenith, $\mathbf{S}$ - south direction, $\mathbf{W}$ - west direction, $\mathbf{P}$ - solar position at time, $\boldsymbol{t}_{\mathbf{0}}, \mathbf{P}^{\prime}$ - the Sun's position at time $\boldsymbol{t}^{\prime}, \mathbf{h}$ - the Sun's altitude at time $\boldsymbol{t}^{\prime}$.

The following paragraph describes how it is possible to calculate the azimuth and the altitude of the Sun on the Moon sky using the proposed model.

Let it be assumed, that the observer is located on $0^{\circ}$ selenographic latitude and on the selenographic longitude $\varphi>0^{\circ}$ (for $\varphi<0^{\circ}$ the problem is symmetrical with respect to the Moon's equator). In this simplified model the rotational axis of the Moon is perpendicular to its orbital plane, hence for a given selenographic longitude the ecliptic great circle intersects the horizon at an angle $90^{\circ}-\varphi$.

At the moment $\boldsymbol{t}_{\mathbf{0}}$ the Sun is located in the maximum elevation equal to $90^{\circ}-\varphi$ (the arc PS). After the time $\boldsymbol{\Delta} \boldsymbol{t}=\boldsymbol{t}^{\prime}-\boldsymbol{t}_{\boldsymbol{0}}$ the Sun will be located in $\mathbf{P}^{\prime}$, and will draw the $\operatorname{arc} \mathbf{P P}^{\prime}=\boldsymbol{\varepsilon}$. At the time $\boldsymbol{t}$ ' solar altitude $\mathbf{h}$ is equal to the $\operatorname{arc} \mathbf{P}$ 'G. The elevation arc is a part of the great circle ZP' $\mathbf{G}$, and can be used for the azimuth (A) estimation, which is equal to arc SG (here it is assumed that the azimuth is counted clockwise from the south direction). Also, the arc $\mathbf{P}^{\prime} \mathbf{W}$ is equal to $90^{\circ}-\varphi$ as a section of the arc PW. Now using the law of sines for the parallactic triangle $\mathbf{P}$ 'GW we have:

$$
\frac{\sin 90}{\sin (90-\varepsilon)}=\frac{\sin (90-\varphi)}{\sin h}
$$

This directly leads to the estimation of the altitude, as:

$$
h=\arcsin (\cos \varphi \quad \cos \varepsilon)
$$

From the law of sines for the parallactic triangle $\mathbf{Z P P}$ ' it is:

$$
\frac{\sin 90}{\sin (90-h)}=\frac{\sin A}{\sin \varepsilon}
$$

Using the formula (9) the azimuth A may be described as:

$$
A=\arcsin \left(\frac{\sin \varepsilon}{\cos h}\right)
$$

With formulae (8) \& (10) available, it is possible to describe the Sun's position in the horizontal coordinate system for a given arc $\varepsilon$. 
For engineering purposes it is not necessary to calculate the Sun's position for the real time $t$, as only general description of the altitude as the function of time and the selenographic latitude is of interest.

It is also possible to perform a simple estimation of the length of the arc $\varepsilon$. The Moon's rotation period $\mathrm{T}_{\mathrm{K}} \approx 27.322$ Eearth's days, and lunar year in our model is identical with Earth's year $\mathrm{P}_{\mathrm{K}} \approx 365.256$ days. If $\Delta \mathrm{t}$ is given in days we can simply estimate $\varepsilon$ using following formula:

$$
\varepsilon=\Delta t\left(1 / T_{K}-1 / P_{K}\right)
$$

as the combination of orbital and rotational motion of the Moon.

Equation (11) may be also simply described as:

$$
\varepsilon=\Delta t\left(1 / T_{S}\right) \cdot 360 / \mathrm{deg} /
$$

where $\mathbf{T}_{\mathbf{S}}$ is so called the Moon's synodic month i.e. the period of lunar phases, of mean value 29.53 days.

In order to verify accuracy of described analytical model, its results were compared with precisely calculated Sun's positions, determined by Stellarium astronomical software [22].

Table 2. Comparison of Sun's position calculated for selected dates by presented analytical model and Stellarium planetarium software. Note the fact, that 03.01 .2018 and 06.07 .2018 are the dates of

Earth's perihelion and aphelion respectively, and 21.03.2018 is the date of March equiniox.

\begin{tabular}{|c|c|c|c|c|c|}
\hline \multirow{2}{*}{ Date and time [UTC] } & \multirow{2}{*}{$\begin{array}{c}\text { Time since local dawn } \\
\text { [days] }\end{array}$} & \multicolumn{2}{|c|}{ Stellarium } & \multicolumn{2}{c|}{ Analytical model } \\
\cline { 2 - 6 } & 0.5 & $\mathbf{H}_{\text {sol }}$ & $\mathbf{A}_{\text {sol }}$ & $\mathbf{H}_{\text {sol }}$ & $\mathbf{A}_{\text {sol }}$ \\
\hline $26.12 .2017,05: 32$ & 7 & 69.30 & 271.85 & 5.73 & 272.09 \\
\hline $01.01 .201817: 32$ & 14 & 10.10 & 864.00 & 69.48 & 346.58 \\
\hline $08.01 .201817: 32$ & 0 & 0.00 & 269.77 & 0.76 & 86.78 \\
\hline $24.03 .2018,12: 02$ & 4.5 & 49.63 & 295.35 & 50.21 & 295.90 \\
\hline $29.03 .2018,00: 02$ & 11.5 & 37.80 & 73.58 & 36.99 & 74.09 \\
\hline $05.04 .2018,00: 02$ & 2.5 & 27.98 & 281.17 & 28.46 & 281.38 \\
\hline $22.07 .2018,10: 22$ & 9 & 62.52 & 45.57 & 62.20 & 46.34 \\
\hline $29.07 .2018,10: 22$ & 13 & 20.62 & 82.13 & 20.17 & 82.32 \\
\hline $02.08 .2018,10: 22$ & & & & & \\
\hline
\end{tabular}

As seen in table 2. There are just minor differences between Calculated positions of the Sun at Moon's sky and Actual values. We consider Such accuracy as fully satisfactory for lunar engineering purposes. This simple astrometric AL model may be easily incorporated into any software, and provide reliable astrometric AL data for energy calculations at the Moon's surface.

\subsection{Solar angle of incidence}

Having the Sun's position on the sky in horizontal coordinate system (see: 2.2. A and H), it is possible to derive a formula to calculate solar angle of incidence for a given plane.

An angle of incidence is an angle between the normal of a given plane and A direction of the incident sunlight.

The plane has the azimuth $A_{P}$, i.e. the angle between the local meridian and the intersection between the plane and the Moon surface, counted clockwise from the South direction. For the model's simplicity we assume, that local Moon's surface normal is perpendicular to the Zenith direction. 
We can simply describe the azimuth of considered plane normal $A_{n}$ :

$$
A_{n}=90-A_{p}
$$

Now, applying the formulae of the spherical trigonometry to the parallactic triangle between the zenith, the Sun's position and the plane's normal elevation $h_{p}$ (i.e. the angle between the normal and the Moon surface) we have:

$$
\cos (\delta)=\cos (90-h) \cdot \cos \left(90-h_{P}\right)+\sin (90-h) \cdot \sin \left(90-h_{p}\right) \cdot \cos \left(A_{n}-A\right)
$$

where $\delta$ is the angle between the normal of a given plane and the Sun's position. From the formula presented above we can calculate $\delta$ as:

$$
\delta=\arccos \left(\sin (h) \cdot \sin \left(h_{p}\right)+\cos (h) \cdot \cos \left(h_{p}\right) \cdot \cos \left(A_{n}-A\right)\right.
$$

\subsection{Program}

In order to conduct considerable amount of necessary calculations and to automate the process, a suitable program was created using MATLAB version 2012A. To obtain the highest possible accuracy of the results, we assumed time step $\Delta \tau=0.001$ days $=1.44$ minutes.

Further decreasing of the time step was found useless.

\section{Results and discussion}

Assuming surface tilt with respect to horizontal plane $\alpha=\mathrm{H}_{\mathrm{w}}-180^{\circ}$, at a specified selenographic latitude $\Phi$, diurnal irradiances at orientation $S_{|\alpha-\Phi|}$, equals diurnal equatorial irradiances at orientation $\mathrm{S}_{\alpha}$.

$\Phi \mathrm{S}_{|\alpha-\Phi|}=0 \mathrm{~S}_{\alpha}$

As horizontal planes at any latitude may be designated as $\Phi \mathrm{S}_{0}$, horizontal surface irradiances at latitude fi is the same as $0 \mathrm{~S}_{\Phi}$.

Considering all this, it was more convenient to depict equatorial southern irradiances for a variety of plane tilts separately, instead of adding several data series for every graph. These equatorial southern irradiances are presented in figure 2.

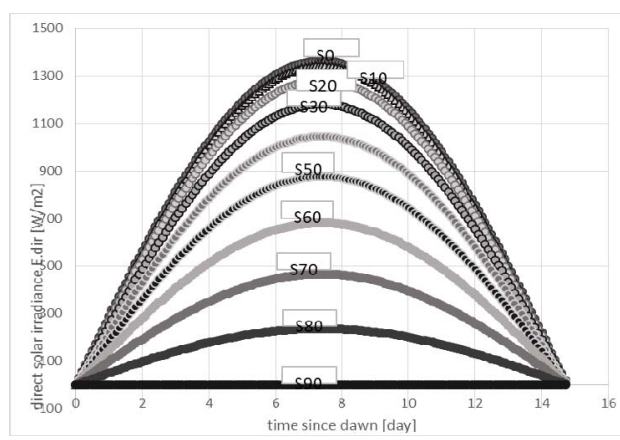

Fig. 2. Irradiances of South-oriented surfaces at the Moon's equator Moreover, because in our model the Sun's positions before and after local noon are exactly symmetrical with respect to the Sun's upper culmination, instantaneous values of irradiance for west and east oriented surfaces are also symmetrical with respect to day 7.38. Thus showing only one of these two were found necessary. The same directional symmetry is to be observed for lunar diurnal insolation's. 

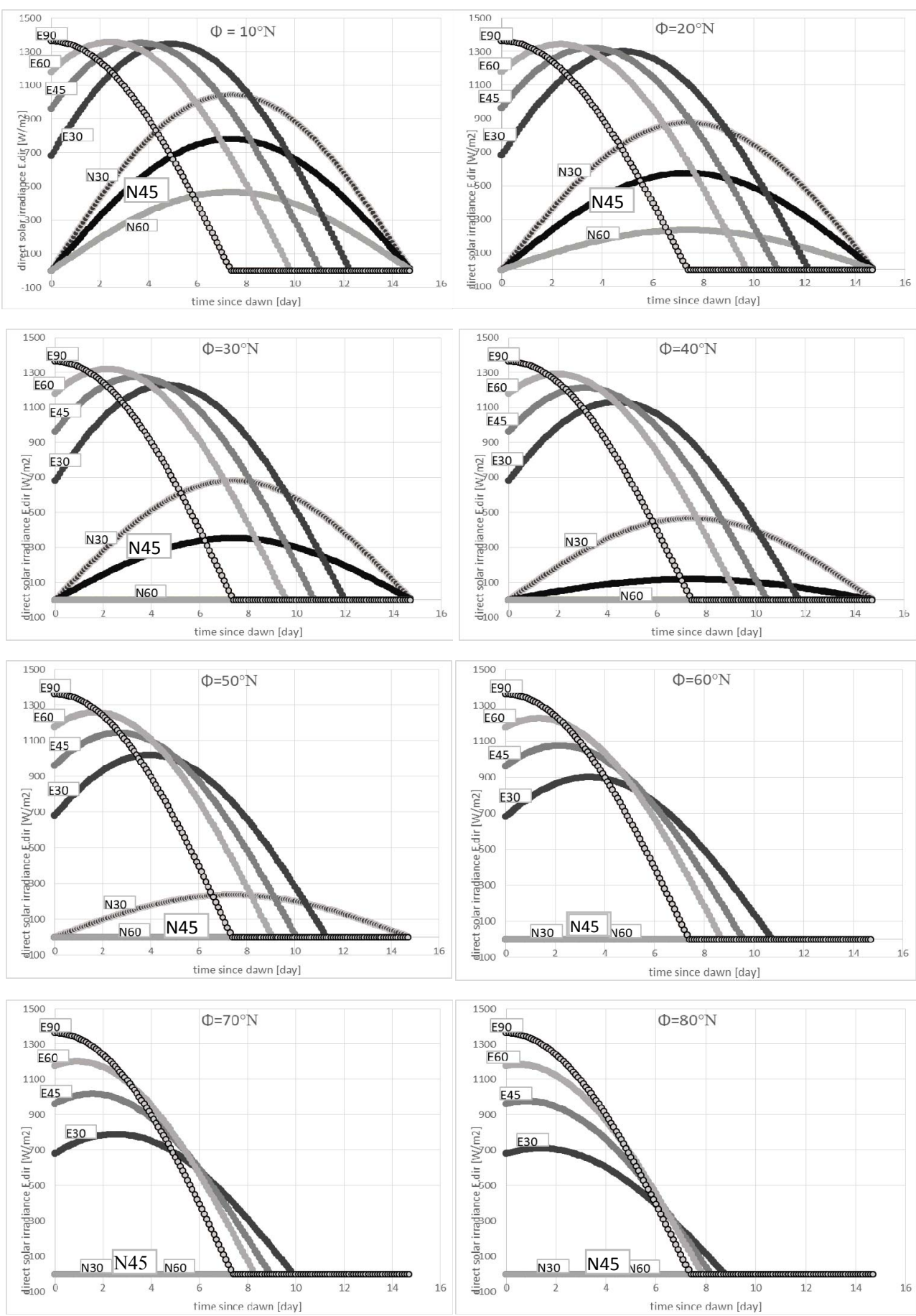

Fig. 3. Diurnal solar irradiances for specified surface orientations at various selenographic latitudes, as a function of lunar daytime. 
for south- oriented surfaces with tilt equal selenographic latitude, noon irradiance reaches the value of lunar TSI. This phenomenon does not occur at the Earth's surface due to sunlight atmospheric attenuation. This phenomenon is exceptionally advantageous for tracking photovoltaic systems. It is easy to calculate, that maximum theoretical insolation for dual axis tracker is $1740 \mathrm{MJ} / \mathrm{m}^{2}$ per lunar day, at any latitude. This value is $157 \%$ of most optimally oriented fixed system, while on Earth, dual-axis solar tracking increases yield of a PV system between 25 and 40\% [20].

Table 3. Lunar diurnal insolations $\left[\mathrm{MJ} / \mathrm{m}^{2}\right]$ for south, north and east-oriented surfaces (columns 2-13). The last column contains the values of total solar energy [MJ] received by reference hemisphere at various latitudes.

\begin{tabular}{|r|r|r|r|r|r|r|r|r|r|r|r|r|r|}
\hline $\begin{array}{r}\text { Seleno- } \\
\text { graphic } \\
\text { latitude }\end{array}$ & $\begin{array}{c}\text { Hori- } \\
\text { zontal }\end{array}$ & S 30 & S 45 & S 60 & S 90 & $\mathbf{N} 30$ & $\mathbf{N ~ 4 5}$ & $\mathbf{N ~ 6 0}$ & $\mathbf{E ~ 3 0}$ & $\mathbf{E} 45$ & $\mathbf{E ~ 6 0}$ & $\mathbf{E}$ 90 & $\begin{array}{c}\text { Reference } \\
\text { hemisphere } \\
\text { [MJ] }\end{array}$ \\
\hline $0^{\circ}$ & 1107 & 958.7 & 782.8 & 553.5 & 0 & 958.7 & 782.8 & 553.5 & 1032.9 & 944.9 & 830.3 & 553.6 & 111759.4 \\
\hline $10^{\circ}$ & 1090.2 & 1040.2 & 906.8 & 711.6 & 192.2 & 848.0 & 634.9 & 378.6 & 1019.3 & 934.8 & 824.0 & 553.6 & 111099.0 \\
\hline $20^{\circ}$ & 1040.2 & 1090.2 & 1003.3 & 848.0 & 378.6 & 711.6 & 467.8 & 192.2 & 979.1 & 904.9 & 805.5 & 553.6 & 109137.7 \\
\hline $30^{\circ}$ & 958. & 1107.0 & 1069.3 & 958.7 & 553.5 & 553.5 & 286.5 & 0 & 914.1 & 856.7 & 775.6 & 553.6 & 105935.3 \\
\hline $40^{\circ}$ & 848.0 & 1090.2 & 1102.8 & 1040.2 & 711.6 & 378.6 & 96.5 & 0 & 827.0 & 792.9 & 736.2 & 553.6 & 101588.9 \\
\hline $50^{\circ}$ & 711.6 & 1040.2 & 1102.8 & 1090.2 & 848.0 & 192.2 & 0 & 0 & 722.3 & 716.9 & 689.2 & 553.6 & 96230.7 \\
\hline $60^{\circ}$ & 553.5 & 958.7 & 1069.3 & 1107.0 & 958.7 & 0 & 0 & 0 & 605.8 & 633.3 & 637.3 & 553.6 & 90023.5 \\
\hline $70^{\circ}$ & 378.6 & 848.0 & 1003.3 & 1090.2 & 1040.2 & 0 & 0 & 0 & 485.6 & 547.5 & 583.3 & 553.6 & 83155.8 \\
\hline $80^{\circ}$ & 192.2 & 711.6 & 906.8 & 1040.2 & 1090.2 & 0 & 0 & 0 & 372.3 & 465.2 & 529.9 & 553.6 & 75836.4 \\
\hline
\end{tabular}

As the proposed astrometric model assumes the Moon's rotational axis perpendicular to the ecliptic plane, insolation at $0 \mathrm{~S}_{90}$ equals 0 , the same as $\Phi \mathrm{N}_{\alpha>90-\Phi}$. In fact, this is not entirely so due to small (but non 0) Moon's axial tilt, not to mention surface diffused radiation [15]. There exists strictly geometrical dependence of both irradiance and insolation values on selenographic latitude and surface tilt. This is mostly due to lack of lunar atmosphere (always 0 atmospheric mass), but also partially due to neglecting surface diffuse radiation For non-directly illuminated surfaces, such as N90, or E90 after local noon. This diffuse radiation would highly influence temperature of indirectly illuminated surfaces, and should be included in future, more advanced studies. Thus, the most reliable comparison would be based on insolations of horizontal surfaces.

Table 4. Horizontal surface monthly insolations $\left[\mathrm{MJ} / \mathrm{m}^{2}\right]$ calculated for 29.531 day period at specified locations on Earth [21].

\begin{tabular}{|c|c|c|c|c|c|}
\hline & $\begin{array}{c}\text { Nairobi, } \\
\text { Kenya } \mathbf{1}^{\circ} \mathbf{1 6}^{\prime} \mathbf{S}\end{array}$ & $\begin{array}{c}\text { New Delhi, } \\
\text { India } \\
\mathbf{2 8}^{\circ} \mathbf{0 7}\end{array}$ & $\begin{array}{c}\text { Bucharest, } \\
\text { Romania } \\
\mathbf{4 4}^{\circ} \mathbf{2 5}^{\prime} \mathbf{N}\end{array}$ & $\begin{array}{c}\text { Warsaw, } \\
\text { Poland } \\
\mathbf{5 2}^{\circ} \mathbf{1 3}^{\prime} \mathbf{N}\end{array}$ & $\begin{array}{c}\text { Oslo, norway } \\
\mathbf{5 9}^{\circ} \mathbf{5 4}^{\prime} \mathbf{N}\end{array}$ \\
\hline $\mathrm{I}_{\mathrm{H} \text { min }}$ & 536.87 & 366.78 & 123.32 & 71.23 & 25.51 \\
\hline $\mathrm{I}_{\mathrm{H} \text { man }}$ & 622.37 & 531.12 & 385.82 & 301.22 & 294.93 \\
\hline $\mathrm{I}_{\mathrm{H} \text { max }}$ & 719.73 & 679.33 & 637.87 & 543.25 & 595.34 \\
\hline
\end{tabular}

comparing data from tables (3) and (4) it is to be observed, that the Moon's insolations at low latitudes are almost twice that of the Earth's. This ratio increases for higher latitudes due to raising impact of earth's atmosphere on incident sunlight for low solar elevation 
angles. Moreover, relatively large Earth's axial tilt causes considerable seasonal variations of monthly insolation values, that are negligible on the Moon.

During a lunar day, 10 metres in diameter reference hemisphere receives a total of $111.75 \mathrm{GJ}$ of solar energy at the equator. As the latitude grows, This value decreases slowly, but with increasing rate, dropping to almost $68 \%$ of the equatorial value at $80^{\circ}$. It is to be observed, that the rate, at which hemispherical insolation decreases is much lower than for any flat surface.

\section{Conclusions}

- The astrometric AL model presented in this paper allows for convenient determination of the Sun's position at lunar sky, as well as to calculate the angle of incidence of solar radiation, with accuracy fully satisfactory for lunar engineering purposes.

- The model was successfully applied to create irradiance and insolation database of direct solar radiation.

- Presented method enables to enhance this database for any selenographic latitude and for any surface orientation.

- The Moon is an exceptionally advantageous place for solar energy exploitation. Such a reliable and powerful energy source would be able to power future lunar habitation and industry centres.

- It is recommended to enhance this model with surface diffused radiation component.

\section{References}

1. Sun Fact Sheet - the NSSDCA - NASA https://nssdc.gsfc.nasa.gov/planetary/factsheet/sunfact.html

2. S. Breiter D. Vokrouhlicky - Mon. Not. R. Astron. Soc. 410, 2807-2816 (2011) Yarkovsky-O'Keefe-Radzievskii-Paddack effect with anisotropic radiation

3. Johnson, F. S., "The Solar Constant"; J. Meteorology, 11(6), 431, 1954.

4. Calculation of monthly average insolation on tilted surfaces SA Klein - 1977 - Sol. Energy , 19(4), 325-432 (1977)

5. Moon Fact Sheet - the NSSDCA - NASA https://nssdc.gsfc.nasa.gov/planetary/factsheet/moonfact.html

6. R.J. Christie, D.W. Plachta, M.M. Hasan, (Transient Thermal Model and Analysis of the Lunar Surface and Regolith for Cryogenic Fluid Storage, in: NASA/TM-2008215300, NASA, Cleveland, 1-13, 2008)

7. A. Vasavada, Joshua L. Bandfield,2 Benjamin T. Greenhagen,1 P. O. R. Hayne, M. A. Siegler, J. P. Williams, D. A. Paige, J. Geophys. Res., 117, E00H18, (2012)

8. LCROSS Impact Data Indicates Water on Moon | NASA https://www.nasa.gov/mission.../LCROSS/.../prelim_water_results

9. M.G. Langseth, S.J. Keihm, In-situ measurements of lunar heat flow, in: Proc. Sov.Am. Conf. Geochem. Moon Planets, NASA SP-370, NASA, Washington, D.C., 283293 (1977)

10. M. G. Langseth, JR. S. P. Clark, JR. .J L. Chute, JR., S.J. Keihm, A. E. Wechsler (The Apollo 15 Lunar Heat-Flow)

11. C. Heinicke, B. Foing, (MaMBA - a functional Moon and Mars Base Analog)

12. I. Srivastava, Space Policy 37 92-96 (2016)

13. L. D. Moondust, Materials Today, 18 (4), (2015) 
14. V. L. Miranda, J.R. Valdes, D.D. Cortes, Constr. Build. Mater., 139 241-246 (2017)

15. Ramesh B. Malla, Kevin M. Brown, Acta Astronaut., 107 196-207 (2015)

16. Haym Benaroyaa, Leonhard : Engineering of lunar bases, Acta Astronaut. 62 277-299 (2008)

17. L. Lichołai, M. Musiał, J. Szyszka Pat. App. EP3091166-A1 (2015)

18. (Overheating Analysis of a Naturally Ventilated Office Building in a Temperate Climate H. Altan, I. Ward, J. Mohelnikova and F. Vajkay PLEA 2008-25th Conference on Passive and Low Energy Architecture, Dublin, 22nd to 24th October 2008)

19. Mohammed Bekhti-In Orbit Irradiation Effects Evaluation of the Alsat-1 Solar Panels International Journal of Energy Science and Engineering 1 (1), 24-30 (2015)

20. Heinrich Häberlin- Photovoltaics: System Design and Practice

21. Solar Electricity Handbook - the irradiance calculator http://solarelectricityhandbook.com/solarirradiance.htmlhttp://solarelectricityhandbook.com/solar-irradiance.html

22. Stellarium Astronomy Software v. 0.18.1 http://stellarium.org/ 